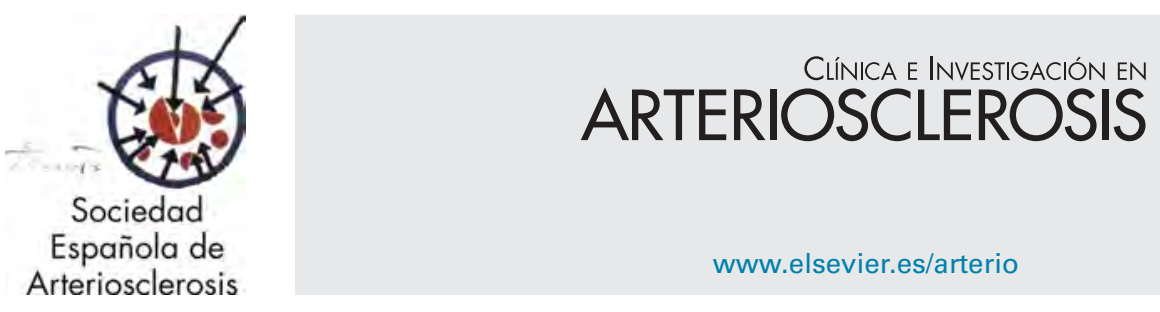

NOTA CLÍNICA

\title{
Tratamiento de un varón con enfermedad de McArdle y muy alto riesgo cardiovascular con inhibidores de PCSK9
}

\author{
Victoria Marco-Benedía,*, Estíbaliz Jarautaa ${ }^{\mathrm{a}, \mathrm{b}}$, Sofía Pérez-Calahorra ${ }^{\mathrm{a}}$, \\ Ana M. Bea ${ }^{a}$ y Fernando Civeira ${ }^{a, b}$ \\ a Unidad Clínica y de Investigación en Lípidos y Arteriosclerosis, Hospital Universitario Miguel Servet, IIS Aragón, CIBERCV, \\ Zaragoza, España \\ b Universidad de Zaragoza, Zaragoza, España
}

Recibido el 25 de julio de 2018; aceptado el 18 de noviembre de 2018

\section{PALABRAS CLAVE \\ Enfermedad de McArdle; Rabdomiólisis; Intolerancia a estatinas; Inhibidores de PCSK9}

\section{KEYWORDS}

McArdle's disease; Rhabdomyolysis; Statin intolerance; PCSK9 inhibitors

\begin{abstract}
Resumen Varón de 60 años con hiperlipidemia familiar combinada, cardiopatía isquémica y diabetes tipo 2. Desde la infancia, intolerancia al esfuerzo intenso. Se le diagnosticó enfermedad de McArdle a raíz de rabdomiólisis asociada a estatinas tras un infarto de miocardio. Desde entonces había seguido tratamiento con dieta, fibratos y ezetimiba con buena tolerancia, pero a pesar de ello las concentraciones de colesterol LDL (cLDL) eran $>180 \mathrm{mg} / \mathrm{dl}$. Se asoció al tratamiento alirocumab $150 \mathrm{mg}$ subcutáneos cada 14 días, con excelente respuesta clínica y descenso de cLDL a $15 \mathrm{mg} / \mathrm{dl}$, manteniéndose estable desde entonces. Nuestro caso demuestra que los inhibidores de PCSK9 son eficaces y seguros en pacientes con enfermedades musculares que contraindican las estatinas y que son una alternativa terapéutica ideal para este tipo de pacientes.

(c) 2018 Sociedad Española de Arteriosclerosis. Publicado por Elsevier España, S.L.U. Todos los derechos reservados.
\end{abstract}

Treatment of a high cardiovascular risk patient with McArdle's disease with PCSK9 inhibitors

Abstract A 60-year-old male with familial combined hyperlipidemia, ischemic heart disease and type 2 diabetes. Since childhood, intolerance to intense exercise. The patient was diagnosed of McArdle's disease after an episode of rhabdomyolysis associated with statins as treatment after a myocardial infarction. Since then, he had been treated with diet, fibrates and ezetimibe with good tolerance, despite this, $\mathrm{LDL}$ cholesterol $(\mathrm{CLDL})$ remained $>180 \mathrm{mg} / \mathrm{dl}$. He started to

\footnotetext{
* Autor para correspondencia.

Correo electrónico: vmarcobenedi@gmail.com (V. Marco-Benedí).
} 
be treated with alirocumab $150 \mathrm{mg} / \mathrm{sc}$ every 14 days, with excellent clinical response and a decrease in CLDL to $15 \mathrm{mg} / \mathrm{dl}$. Our case shows that PCSK9 inhibitors are effective and safe in patients with muscle diseases who have statin contraindication, and they are a good therapeutic tool for these patients

(c) 2018 Sociedad Española de Arteriosclerosis. Published by Elsevier España, S.L.U. All rights reserved.

\section{Introducción}

La enfermedad de McArdle, también conocida como glucogenosis tipo $\mathrm{V}$, enfermedad de almacenamiento de glucógeno tipo $\mathrm{V}$ o déficit de miofosforilasa, es una enfermedad rara que produce dolores musculares ante pequeños esfuerzos $^{1,2}$. La enfermedad de McArdle es una enfermedad autosómica recesiva y los pacientes afectos presentan mutaciones en ambos alelos del gen PYGM que codifica para la miofosforilasa. Se han identificado más de 65 mutaciones en el gen PYGM causantes de enfermedad de McArdle hasta la actualidad ${ }^{2,3}$. La miofosforilasa inicia la degradación de glucógeno en el músculo, como consecuencia del déficit de actividad de esta enzima. Las personas que la padecen tienen dificultad en obtener la energía de las reservas de glucógeno ${ }^{1,2}$. La ingestión de hidratos de carbono complejos (vegetales, frutas, cereales, pan, pasta y arroz) antes del ejercicio y una proporción del $20 \%$ de grasa en la ingesta total diaria parecen mejorar la tolerancia ${ }^{2}$. No se conoce una cura definitiva hasta el momento, y para aquellos pacientes con diagnóstico de dislipidemia, que además tienen cardiopatía isquémica, se plantean nuevos tratamientos que consigan los objetivos terapéuticos en estos pacientes de alto riesgo. Es por ello que los anticuerpos monoclonales anti-PCSK9 sirvan como terapéutica alternativa para estos pacientes.

\section{Material y métodos}

Presentamos el caso de un varón de 60 años de muy alto riesgo cardiovascular con antecedente de rabdomiólisis con estatinas que acudió por primera vez a nuestra consulta de la Unidad de Lípidos del Hospital Universitario Miguel Servet de Zaragoza, aproximadamente hace 16 años. Fue derivado por su médico de atención primaria para el estudio de su dislipidemia con cifras en ayunas de colesterol total en torno a $300 \mathrm{mg} / \mathrm{dl}$ y $534 \mathrm{mg} / \mathrm{dl}$ de triglicéridos. Diagnosticado de diabetes tipo 2 desde hacía unos 6 años.

Desde la infancia refería mialgias e intolerancia al ejercicio. En varias ocasiones presentó concentraciones elevadas de la enzima creatina cinasa (CPK) en reposo y episodios de mioglobinuria. A los 52 años tuvo un infarto de miocardio. A las pocas semanas, un cuadro de rabdomiólisis con hematuria, mialgias intensas y cifras de CPK > 10.000 UI/l que aparecieron a las 2 semanas de iniciar tratamiento con atorvastatina $20 \mathrm{mg}$ / día.
La primera vez que acudió a nuestra consulta, presentaba astenia severa desde hacía varios meses y el examen físico era normal, con un peso de $81,7 \mathrm{~kg}$ y un índice de masa corporal (IMC) de $27,2 \mathrm{~kg} / \mathrm{m}^{2}$. No tenía arco corneal, ni xantomas. Mostraba telangiectasias malares. Seguía una dieta estricta, pobre en grasa saturada y rica en hidratos de carbono complejos y tratamiento farmacológico con colestipol $5 \mathrm{~g}$ al día desde hacía 4 años. A pesar de ello, su perfil lipídico mostraba: colesterol total $267 \mathrm{mg} / \mathrm{dl}$, triglicéridos $189 \mathrm{mg} / \mathrm{dl}$, colesterol HDL (cHDL) $36 \mathrm{mg} / \mathrm{dl}$, colesterol LDL (cLDL) 193,2 mg/dl, apolipoproteína B (apoB) $186 \mathrm{mg} / \mathrm{dl}$. Las enzimas hepáticas estaban aumentadas: GGT $82 \mathrm{U} / \mathrm{l}$, GPT $82 \mathrm{U} / \mathrm{l}$, GOT $53 \mathrm{U} / \mathrm{l}$ y también presentaba aumento de CPK $4.505 \mathrm{U} / \mathrm{l}$ y de lactato deshidrogenasa (LDH) $523 \mathrm{U} / \mathrm{l}$. El resto de parámetros bioquímicos, incluyendo hormonas tiroideas, glucosa y creatinina, estaban dentro de la normalidad. Ante la sospecha de enfermedad muscular primaria, la biopsia muscular confirmó el diagnóstico de enfermedad de McArdle, enfermedad de almacenamiento de glucógeno tipo V.

Se recomendó iniciar tratamiento con fenofibrato $145 \mathrm{mg}$ al día y se duplicó la dosis de colestipol a $10 \mathrm{~g}$ al día; así mismo, se le indicó que debía limitar su actividad física. La nutricionista reforzó la importancia de seguir una dieta rica en hidratos de carbono complejos, limitación en el consumo de grasas saturadas, de azúcares simples y alcohol, consumo de pescado azul al menos una vez por semana y nueces (al menos 3 unidades) diariamente. En la siguiente visita, un año después, había perdido $5 \mathrm{~kg}$ de peso, pero mantenía la sensación de cansancio y dolores musculares. Las cifras de CPK siguieron aumentadas con 3.000 U/l, el cLDL era de $212 \mathrm{mg} / \mathrm{dl}$ y la hemoglobina glucosilada (HbAc1) del $6,3 \%$. En ese momento se añadió al tratamiento ezetimiba $10 \mathrm{mg}$. Fue revisado periódicamente por nuestra Unidad, sin mostrar cambios substanciales en el examen físico o en el perfil lipídico.

\section{Resultados}

Debido a que el paciente se encontraba muy lejos de los objetivos de $C L D L$ - que al ser de muy alto riesgo son inferiores a $70 \mathrm{mg} / \mathrm{dl}-$, y con la posibilidad de prescripción de inhibidores de proproteína convertasa subtilisina/kexina tipo 9 (PCSK9), el paciente comenzó a principios de 2017 con alirocumab $150 \mathrm{mg}$ subcutáneo cada 14 días, manteniendo ezetimiba $10 \mathrm{mg}$ al día y fenofibrato $145 \mathrm{mg}$ al día, y suspendió colestipol de forma voluntaria por molestias digestivas. 


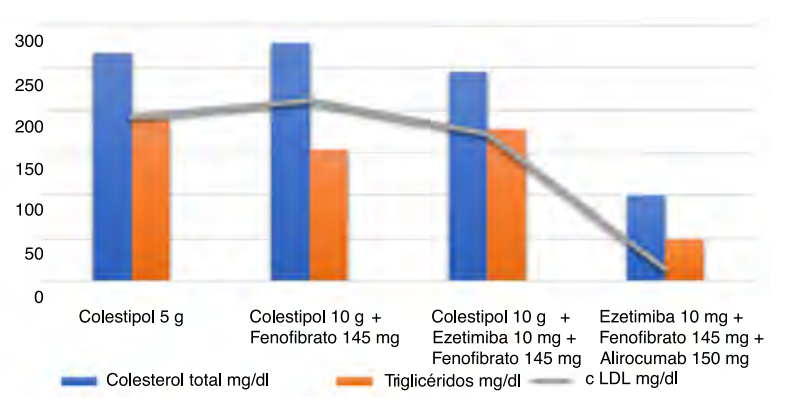

Figura 1 Evolución del perfil lipídico con los diferentes tratamientos.

CLDL: colesterol ligado a lipoproteínas de baja densidad.

No presentó cambios clínicos, permaneciendo sin angina, haciendo vida normal y tolerando el ejercicio ligero diariamente. Las cifras de CPK se han mantenido entre 1.212 y $6.000 \mathrm{U} / \mathrm{l}$, las enzimas hepáticas han permanecido normales y ha habido un llamativo descenso del cLDL con cifras de $15 \mathrm{mg} / \mathrm{dl}$. Su último perfil analítico muestra: colesterol total $100 \mathrm{mg} / \mathrm{dl}$, triglicéridos $175 \mathrm{mg} / \mathrm{dl}$, cHDL $50 \mathrm{mg} / \mathrm{dl}$, CLDL $15 \mathrm{mg} / \mathrm{dl}$, apoB 41,4 mg/dl, HbAc1 6,6\%. Enzimas: GGT $28 \mathrm{U} / \mathrm{l}$, GPT $51 \mathrm{U} / \mathrm{l}$, GOT $47 \mathrm{U} / \mathrm{l}$, CPK $1.212 \mathrm{U} / \mathrm{l}$, LDH $222 \mathrm{U} / \mathrm{l}$. En la actualidad, se mantiene dicho tratamiento (fig. 1).

\section{Discusión}

El caso presentado muestra un paciente con enfermedad de McArdle que mejora la concentración de CLDL con inhibidores de PCSK9 y con excelente tolerancia clínica.

La enfermedad de McArdle es una enfermedad hereditaria autosómica recesiva, causada por el déficit de la miofosforilasa muscular, una enzima muscular encargada de la degradación de glucógeno muscular esquelético ${ }^{4}$. Está caracterizada por astenia, debilidad muscular, calambres, mialgias, intolerancia al ejercicio, además de niveles altos de CPK en reposo y episodios de mioglobinuria, especialmente tras esfuerzos ${ }^{1,3}$.

Este caso es la primera descripción en la literatura de una evolución favorable de la dislipidemia en esta enfermedad y muestra que los inhibidores de PCSK9 pueden servir de tratamiento para estos pacientes. Las estatinas suelen empeorar los síntomas musculares en pacientes con miopatías y por ello se recomienda evitarlas o, en su caso, utilizar dosis bajas y realizar un seguimiento estricto ${ }^{1,5}$. Nuestro paciente tenía episodios de mialgias y malestar cuando realizaba ejercicio que empeoraron en tratamiento con estatinas, incluso desarrollando un episodio de rabdomiólisis grave. Sin embargo, con los inhibidores de PCSK9 hubo una favorable mejoría en cuanto a su perfil lipídico y enzimático sin modificar su clínica muscular. A pesar de las concentraciones muy bajas de CLDL que se han obtenido en este paciente, las dosis de hipolipemiantes se mantuvieron en vista de los excelentes resultados de tolerabilidad y beneficio clínico de las concentraciones bajas de LDL con inhibidores de PCSK9 ${ }^{6}$.

La concentración del receptor LDL en la superficie hepática está controlada por la proteína PCSK9 ${ }^{7}$. Dicha proteína reduce la captación de partículas $L D L$, lo que lleva a un aumento en las concentraciones de CLDL plasmático. Los anticuerpos monoclonales actúan inhibiendo la unión de PCSK9 al receptor LDL. Estos han mostrado disminuciones dependientes de la dosis en el cLDL (44-65\%), apoB (48-59\%) y lipoproteína(a) (27-50\%), sin efectos adversos importantes, incluidos pacientes con intolerancia a las estatinas ${ }^{8}$. En el estudio Odyssey Alternative, 314 pacientes con intolerancia a las estatinas fueron aleatorizados a recibir alirocumab $75 \mathrm{mg}$ cada 2 semanas, ezetimiba $10 \mathrm{mg} /$ día o atorvastatina $20 \mathrm{mg} /$ día. Si no se lograban los objetivos de cLDL, se aumentaba alirocumab a $150 \mathrm{mg}$ cada 2 semanas. Los efectos secundarios a nivel muscular fueron menos frecuentes con alirocumab que con atorvastatina ${ }^{9}$.

Estos hallazgos implican que los inhibidores de PCSK9 podrían ser una alternativa prometedora para reducir el cLDL en pacientes con contraindicación de estatinas ${ }^{10}$.

\section{Conclusiones}

La enfermedad de McArdle es una miopatía causada por el déficit de la miofosforilasa muscular. Estos pacientes experimentan crisis agudas musculares después del ejercicio intenso. En la actualidad no tiene tratamiento y se recomienda una dieta rica en hidratos de carbono complejos y limitación del ejercicio intenso. El tratamiento con estatinas está contraindicado, por lo que en presencia de alto riesgo vascular y concentraciones elevadas de CLDL los inhibidores de PCSK9 son una alternativa terapéutica como demuestra nuestro caso.

\section{Conflicto de intereses}

FC: ha recibido compensaciones económicas de Amgen y Sanofi por conferencias y comités de asesoramiento. Victoria Marco-Benedí y Estíbaliz Jarauta han recibido de Sanofi becas de viaje para asistencia a congresos.

\section{Agradecimientos}

Este caso ha sido premiado por la Sociedad Española de Arteriosclerosis (SEA) en el Congreso Nacional de la SEA celebrado en Gerona en junio de 2018.

\section{Bibliografía}

1. Quinlivan R, Buckley J, James $M$, Twist A, Ball S, Duno M, et al. McArdle disease: a clinical review. J Neurol Neurosurg Psychiatry. 2010;81:1182-8, http://dx.doi.org/10.1136/jnnp. 2009.195040.

2. Leite A, Oliveira N, Rocha M. McArdle disease: a case report and review. Int Med Case Rep J. 2012;5:1-4, http://dx.doi.org/10. 2147/IMCRJ.S28664.

3. Andreu A, Nogales-Gadea G, Cassandrini D, Arenas J, Bruno C. McArdle disease: molecular genetic update. Acta Myol. 2007;26:53-7. PMCID: PMC2949323.

4. Özen H. Glycogen storage diseases: new perspectives. World J Gastroenterol. 2007;13:2541-53, http://dx.doi.org/10. 3748/wjg.v13.i18.2541. 
5. Stroes ES, Thompson PD, Corsini A, Vladutiu GD, Raal FJ, Ray KK, et al. Statin-associated muscle symptoms: impact on statin therapy. Eur Heart J. 2015;36:1012-22, http://dx.doi.org/ 10.1093/eurheartj/ehv043.

6. Giugliano RP, Pedersen TR, Park JG, de Ferrari GM, Gaciong $Z A$, Ceska R, et al. Clinical efficacy and safety of achieving very low LDL-cholesterol concentrations with the PCSK9 inhibitor evolocumab: a prespecified secondary analysis of the FOURIER trial. Lancet. 2017;390(10106):1962-71, http://dx.doi.org/10.1016/S0140-6736(17)32290-0.

7. Schulz R, Schlüter KD, Laufs U. Molecular and cellular function of the proprotein convertase subtilisin/kexin type 9 (PCSK9). Basic Res Cardiol. 2015;110:4, http://dx.doi.org/10. 1007/s00395-015-0463-z.

8. Bergeron N, Phan BA, Ding $Y$, Fong A, Krauss RM. Proprotein convertase subtilisin/kexin type 9 inhibition: a new therapeutic mechanism for reducing cardiovascular disease risk. Circulation. 2015;132:1648-66, http://dx.doi.org/ 10.1161/CIRCULATIONAHA.115.016080.

9. Moriarty PM, Jacobson TA, Bruckert E, Thompson PD, Guyton JR, Baccara-Dinet MT, et al. Efficacy and safety of alirocumab, a monoclonal antibody to PCSK9, in statin-intolerant patients: design and rationale of Odyssey Alternative, a randomized phase 3 trial. J Clin Lipidol. 2014;8:554-61, http://dx.doi.org/10.1016/j.jacl.2014.09.007.

10. Stroes E, Colquhoun D, Sullivan D, Civeira F, Rosenson RS, Watts GF, et al. Anti-PCSK9 antibody effectively lowers cholesterol in patients with statin intolerance: the Gauss-2 randomized, placebo-controlled phase 3 clinical trial of evolocumab. J Am Coll Cardiol. 2014;63:2541-8, http://dx.doi.org/10.1016/j.jacc.2014.03.019. 